\title{
Electrophysiological and fundoscopic detection of intracranial hypertension in craniosynostosis
}

\author{
Sohaib R. Rufai ${ }^{1,2,3,4,6}$, Oliver R. Marmoy (D) ${ }^{1,2,6}$, Dorothy A. Thompson (iD ${ }^{1,2}$, Lara S. van de Lande ${ }^{2,4}$, R. William Breakey ${ }^{2,4}$, \\ Catey Bunce (D) $^{5}$, Vasiliki Panteli ${ }^{1}$, Kemmy Schwiebert ${ }^{1}$, Shafquet Mohamed ${ }^{1}$, Frank A. Proudlock ${ }^{3}$, Irene Gottlob ${ }^{3}$, David J. Dunaway ${ }^{2,4}$, \\ Richard Hayward ${ }^{2,4}$, Richard Bowman (iD ${ }^{1,2}$ and Noor ul Owase Jeelani (iD ${ }^{2,4}$ 压
}

(c) The Author(s) 2021

\begin{abstract}
AIMS: To assess the diagnostic accuracy of fundoscopy and visual evoked potentials (VEPS) in detecting intracranial hypertension (IH) in patients with craniosynostosis undergoing spring-assisted posterior vault expansion (sPVE).

METHODS: Children with craniosynostosis undergoing SPVE and 48-hour intracranial pressure (ICP) monitoring were included in this single-centre, retrospective, diagnostic accuracy study. Data for ICP, fundoscopy and VEPs were analysed. Primary outcome measures were papilloedema on fundoscopy, VEP assessments and IH, defined as mean ICP $>20 \mathrm{mmHg}$. Diagnostic indices were calculated for fundoscopy and VEPs against IH. Secondary outcome measures included final visual outcomes.

RESULTS: Fundoscopic examinations were available for 35 children and isolated VEPs for 30 children, 22 of whom had at least three serial VEPs. Sensitivity was $32.1 \%$ for fundoscopy (95\% confidence intervals [Cl]: $15.9-52.4$ ) and $58.3 \%$ for isolated VEPs (95\% Cl 36.6-77.9). Specificity for IH was $100 \%$ for fundoscopy (95\% Cl: $59.0-100)$ and $83.3 \%$ for isolated VEPs (95\% Cl: 35.9-99.6). Where longitudinal deterioration was suspected from some prVEPs but not corroborated by all, sensitivity increased to $70.6 \%$ ( $95 \% \mathrm{Cl}$ : 44.0-89.7), while specificity decreased to $60 \%$ (95\% Cl: 14.7-94.7). Where longitudinal deterioration was clinically significant, sensitivity decreased to $47.1 \%$ (23.0-72.2) and specificity increased to $100 \%$ (47.8-100). Median final BCVA was 0.24 logMAR $(n=36)$. UK driving standard BCVA was achieved by 26 patients $(72.2 \%)$, defined as $\geq 0.30$ logMAR in the better eye.

CONCLUSION: Papilloedema present on fundoscopy reliably indicated $\mathrm{IH}$, but its absence did not exclude IH. VEP testing boosted sensitivity at the expense of specificity, depending on method of analysis.
\end{abstract}

Eye (2023) 37:139-145; https://doi.org/10.1038/s41433-021-01839-w

\section{INTRODUCTION}

Craniosynostosis is characterised by the premature fusion of the cranial sutures. Its complex forms are commonly associated with intracranial hypertension $(\mathrm{IH})$ that, if left untreated, can cause cognitive impairment, visual impairment and even death [1]. The estimated prevalence of craniosynostosis is 3.1-6.4 in 10,000 births and rising [2]. It can be sub-classified as non-syndromic (approximately one-third of cases) that affects one or multiple sutures, or syndromic (approximately two-thirds of cases), the majority of whom have multiple suture fusions often combined with extracranial anomalies [3]. IH occurs in $30-40 \%[4,5]$ of the syndromic cases, most commonly in patients diagnosed with Apert (71\%) [6], Crouzon (61\%) [7] and Pfeiffer (60\%) [8] syndromes. In the non-syndromic cases, IH occurs in approximately $17 \%$ [9] with a single fused suture and in $24-47 \%$ [1, 8] with multiple sutures involved.

Surgical management of $\mathrm{IH}$ primarily involves expansion of the skull vault, once hydrocephalus has been excluded. This reduces the risk of associated sequelae by increasing the intracranial volume. It also allows some correction of a brachycephalic skull shape. At Great Ormond Street Hospital for Children (GOSH), the procedure of choice is a spring-assisted posterior vault expansion (SPVE) [10]. It is a less invasive operation than a fronto-orbital reconstruction, providing a greater degree of vault expansion [11] and leaving the frontal region intact for any subsequent fronto-facial surgery.

Detecting $\mathrm{IH}$ in children is challenging. Direct intracranial measurement of intracranial pressure (ICP) is the gold standard, but this involves hospital admission and general anaesthesia as well as the risks of infection, bleeding, cerebrospinal fluid leakage and mechanical failure [5]. Non-invasive ophthalmological methods of detecting ICP employed at GOSH include fundoscopy [12] and visual evoked potentials (VEPs) [13], performed according to a surveillance protocol [14]. The patient's visual acuity (VA), comprehensive neurosurgical evaluation and radiological findings [15] are also considered but, when concerns remain, 48-h ICP monitoring is performed [16].

\footnotetext{
${ }^{1}$ Clinical and Academic Department of Ophthalmology, Great Ormond Street Hospital for Children NHS Foundation Trust, London WC1N 3JH, UK. ${ }^{2}$ UCL Great Ormond Street Institute of Child Health, London WC1N 1EH, UK. ${ }^{3}$ The University of Leicester Ulverscroft Eye Unit, Leicester Royal Infirmary, Robert Kilpatrick Clinical Sciences Building, PO Box 65, Leicester LE2 7LX, UK. ${ }^{4}$ Craniofacial Unit, Great Ormond Street Hospital for Children NHS Foundation Trust, London WC1N 3JH, UK. ${ }^{5} \mathrm{Clinical}$ Trials Unit, The Royal Marsden NHS Foundation Trust, London SW3 6JJ, UK. ${ }^{6}$ These authors contributed equally: Sohaib R. Rufai, Oliver R. Marmoy. Presentation This work was presented in part as an e-Poster at the virtual Association for Research in Vision and Ophthalmology (ARVO) Annual Meeting, 4 May 2021, and won the ARVO Foundation Travel Award. email: Owase.Jeelani@gosh.nhs.uk
} 
The primary objective of this study was to assess the diagnostic accuracy of ophthalmological monitoring methods (fundoscopy and VEPs) in detecting $\mathrm{IH}$ in a cohort of children with craniosynostosis who underwent sPVE after ICP monitoring. Our secondary objective was to evaluate visual outcomes.

\section{MATERIALS AND METHODS \\ Study design and participants}

This was a diagnostic accuracy study of patients with craniosynostosis undergoing ICP assessment and SPVE surgery at GOSH-a quaternary paediatric referral centre in the United Kingdom. Data were collected retrospectively between 16 February 2002 and 12 March 2019, to capture all children who underwent sPVE since the technique was started at GOSH. This study is reported according to the Standards for Reporting of Diagnostic Accuracy Studies (STARD) guidelines [17]. This study adhered to the tenets of the Declaration of Helsinki. Ethical approval was obtained for this study (UK REC 15/LO/0386-Research Ethics Committee approvalStudy No. 14DS25).

Inclusion criteria were as follows: (i) children diagnosed with craniosynostosis undergoing sPVE surgery; (ii) availability of 48-h ICP assessment(s); (iii) availability of fundoscopic examination and/or VEP assessment within at least 6 months of the ICP assessment. Wherever children had three serial VEP assessments, where the most recent fell within 6 months of the ICP assessment, these were also included in the diagnostic accuracy testing. Children with fundoscopic examinations and/or VEP assessments more than 6 months prior to their ICP assessment were excluded from diagnostic accuracy testing, as those exceeding this time-frame were unlikely to be reflective of true change. Adults aged 18 or over were excluded.

The following baseline characteristics were recorded: diagnosis, gender, age at first presentation at GOSH, age at first sPVE surgery, age at first bolt ICP assessment, age at final follow-up, total follow-up and final destination. Diagnoses were based on genetic testing, radiological findings and clinical assessment. Final destination was defined as further follow-up at GOSH, transfer to local hospital or discharge to community opticians.

\section{Primary outcome measures}

The primary outcome measures were papilloedema on fundoscopy, VEP assessments and 48-h ICP assessments. Diagnostic accuracy was assessed for fundoscopy and VEP against IH (present/absent).

Fundoscopy. Fundoscopy was performed by experienced paediatric neuro-ophthalmologists (RB and VP). Examinations immediately prior to ICP assessment and at final visit were recorded. Optic disc examinations were graded from the clinical notes as 'normal', 'swollen', 'mild pallor' or 'atrophic'. Frequency of fundoscopic examinations was as per our surveillance protocol [14]; only examinations within 6 months of bolt ICP examination were included.

VEP methods and analysis. Pattern reversal VEPs (prVEPs) were recorded to high-contrast black and white reversing checkerboards presented on a plasma display panel subtending a $30^{\circ}$ field of mean luminance $82 \mathrm{~cd} / \mathrm{m}^{2}$ in accordance with International Society for Clinical Electrophysiology of Vision (ISCEV) standards [18]. Check widths presented with both eyes open ranged between $200^{\prime}$ and $6.25^{\prime}$ depending on the age, ability and normality of responses. $\mathrm{Ag}-\mathrm{AgCl}$ electrodes were applied with conductive paste over the occipital scalp at $\mathrm{Oz}$, with a reference electrode placed at $\mathrm{Fz}$ and ground at $\mathrm{Cz}$. Signals were filtered between 0.3 and $100 \mathrm{~Hz}$ with a minimum of 50 trials obtained in a minimum of two averages, unless patient cooperation limited this. All data were acquired with an Espion $\mathrm{E}^{3}$ system (Diagnosys LLC, Cambridge, UK).

VEPs were scheduled in accordance with our surveillance protocol [14]. Two independent reviewers (ORM and DAT), masked to the other outcome measures including fundoscopy and ICP, retrospectively analysed and graded the prVEPs in two ways. The first was an analysis of prVEPs to a single check width (50') from the recording closest to, and within 6 months of the ICP bolt measure. This 'isolated' single check, single episode analysis allows comparison with previously published data and the ISCEV VEP standard [18].

In this 'isolated' analysis, prVEP abnormality was determined relative to the $95 \%$ laboratory reference ranges for the ISCEV large check, which in this study were a latency of $90-116 \mathrm{~ms}$ and an amplitude of 5-62 $\mu \mathrm{V}$. Measured prVEPs were graded using the criteria created by Thompson et al. [13]. This published prVEP grading system for $50^{\prime}$ check widths was modified slightly to reclassify grade 3 as 'abnormal amplitude' rather than 'reduced amplitude', and grade 4 as 'abnormal peak-time' rather than 'delayed peak-time'. This allowed us to account for prVEPs which were atypically large (i.e., 'giant VEPs', $n=1$ ) exceeding the reference limit $(>62 \mu \mathrm{V})$ and those of atypically early peak times $(n=1)$ earlier than reference limits $(>90 \mathrm{~ms}$ ), which suggests a paramacular dominance of the VEP with reduced central field sensitivity (Table 1). For the sensitivity/ specificity analysis, VEP grades 1 or 2 were considered normal whilst grades 3-6 were considered abnormal as their values fell outside the laboratory reference range for amplitude and/or peak-time.

The second analysis undertaken was a longitudinal inspection of prVEPs to a range of check widths $\left[200^{\prime}, 100^{\prime}, 50^{\prime}, 25^{\prime}, 12.5^{\prime}\right.$ and $\left.6.25^{\prime}\right]$ recorded in three successive appointments prior to the ICP assessment, the closest within 6 months of the ICP bolt. The longitudinal stability of prVEPs was graded as follows: $0=$ stable, $1=$ equivocal, $2=$ deterioration and $-1=$ improvement. A clinically significant deterioration was regarded if a cumulative score over visits was $\geq 2$, a subtle/suspicious deterioration if the cumulative score was $\geq 1$.

The consideration of 'stability' for patients with craniosynostosis included prVEP data produced to all check widths. Changes in measured amplitude, peak-time and waveform morphology across visits were noted. In cases where prVEPs were degraded (i.e., grades $5 / 6$ ), stability was determined using pattern onset or flash VEPs when recorded. 'Equivocal' prVEPs are those suspicious of deterioration to some check widths, but not corroborated across all checks, and include a qualitative assessment of test compliance within the quantitative change. It is summarised broadly as follows:

- A P100 amplitude change of $\geq 30 \%$ in no more than 1-2 check widths

- A latency change of $>5$ ms of P100 to 1-2 check widths (typically small checks-12.5'/6.25')

- A change of $>50 \%$ in amplitude or latency across $\geq 2$ check widths, but with sub-optimal prVEP test compliance

ICP assessment. Patients were admitted for gold standard, 48-hr ICP assessment as inpatients following ophthalmological monitoring. The RAUMEDIC ICP bolt (RAUMEDIC AG, Helmbrechts, Germany) was used. ICP was assessed over 48-hr and reported by a consultant specialising in ICP sleep studies and/or experienced consultant craniofacial surgeons (NuOJ and DJD), all of whom had access to the ophthalmological monitoring results as per the usual GOSH clinical policy. We considered mean ICP values between 10 and $20 \mathrm{mmHg}$ as within the normal range in children with

Table 1. VEP grading criterion modified from Thompson et al. (2006) [13].

\begin{tabular}{ll} 
VEP grade & Modified VEP grading criteria \\
\hline Grade 1 & Normal VEP (amplitude and latency within normal limits) \\
\hline Grade 2 & Normal amplitude and latency, but broadened waveform \\
\hline Grade 3 & Abnormal amplitude (low amplitude or atypically 'giant') with normal latency \\
\hline Grade 4 & Normal amplitude with abnormal latency (prolonged or atypically early latency) \\
Grade 5 & Abnormal amplitude and latency \\
\hline Grade 6 & No VEP response \\
\hline
\end{tabular}

VEP visual evoked potentials. 
craniosynostosis $[16,19]$. Mean ICP values $\geq 20 \mathrm{mmHg}$ were considered raised and classified as $\mathrm{IH}$.

\section{Secondary outcome measures}

Our secondary outcome measures were final BCVA and the proportion of children achieving UK driving standard BCVA $(\leq 0.30$ logMAR) [20]. Final BCVA was measured at final visit using Thomson LogMAR test charts (Thomson Software Solutions, Hatfield, UK) wherever possible; if not possible, then BCVA was tested using forced preferential looking using Keeler Acuity Cards (Keeler Ltd, Windsor, UK), wherever possible.

\section{Additional outcome measures}

Prevalence of amblyogenic risk factors was reported. Amblyogenic risk factors were defined as per the American Association of Pediatric Ophthalmology and Strabismus Guidelines [21].

\section{Statistical analysis}

Sensitivity, specificity, positive predictive value, negative predictive value and accuracy were calculated for fundoscopy and VEPs against IH (present/ absent) based on initial ICP assessment. Accuracy is defined as the proportion of true test results (either true positives or true negatives) amongst all evaluated cases. Calculation of sample size was not applicable given the retrospective nature of this study, rather all eligible children were included to maximise power and avoid selection bias.

\section{RESULTS}

\section{Baseline characteristics}

Table 2 displays the baseline demographics of this cohort. No patients were lost to follow-up. At final visit, 13 patients (31.1\%) had their care transferred from GOSH to local hospitals, while 4 patients (10.8\%) were discharged into community optician care.

\section{Diagnostic accuracy testing}

Prevalence of $\mathrm{IH}$ was $80.0 \%(n=28 ; 95 \%$ confidence interval $[\mathrm{Cl}]$ : 63.1-91.6\%) based on ICP assessments available in 35 patients included in diagnostic accuracy testing. Figure 1 displays the STARD patient flowchart for our diagnostic accuracy testing.

Fundoscopic examinations during appointments within the prior 6 months of first ICP assessments were available in 35 patients. Median time between pre-ICP fundoscopy and first ICP assessment was 1.7 months (IQR: 2.1). Baseline VEP data were available for 35 patients; 30 recordings were within 6 months of the ICP measurement and three serial VEP studies available for 22 patients. Median time between most recent VEP and first ICP assessment was 1.5 months (IQR: 2.3). Table 3 displays $2 \times 2$ contingency tables and diagnostic accuracy testing of fundoscopy and VEPs against IH. Figure 2 displays prVEP waveforms from two sample patients demonstrating deterioration over time. Supplementary Table 1 (Supplementary Material) displays raw VEP data.

Out of the nine patients with papilloedema on fundoscopy prior to ICP assessment, all disc swelling had resolved on final examination. One of these nine patients was noted to have mild bilateral disc pallor, while another had mild unilateral disc pallor on final examination. None of these nine patients developed optic atrophy.

\section{Secondary outcome measures: visual outcomes}

Final visual outcomes were available for 36 out of 37 children (97.3\%). Final BCVA data were recorded by logMAR chart vision testing in 32 children (88.9\%); 3 children (8.3\%) who could not participate with chart testing had forced preferential looking, while 1 child (2.8\%) had perception of light only (logMAR equivalent: 2.70) [22]. Data were unavailable for one child as they were an international patient transferred back to their home country before final ophthalmological examination and BCVA.

Median final BCVA was 0.24 logMAR (IQR: 0.51; range: -0.06 to 2.7). UK driving standard BCVA was achieved by 26 patients (72.2\%),
Table 2. Baseline demographics.

\begin{tabular}{|c|c|c|c|}
\hline $\begin{array}{l}\text { Baseline } \\
\text { characteristics }\end{array}$ & $\begin{array}{l}\text { Number } \\
\text { of } \\
\text { patients }\end{array}$ & Median (IQR) & Range \\
\hline \multicolumn{4}{|l|}{ Diagnosis } \\
\hline \multicolumn{4}{|l|}{ Syndromic } \\
\hline Crouzon & 8 & \multirow[t]{10}{*}{-} & \multirow[t]{10}{*}{-} \\
\hline Apert & 5 & & \\
\hline Pfeiffer & 4 & & \\
\hline ERF & 1 & & \\
\hline Muenke & 1 & & \\
\hline Smith-Lemli-Opitz & 1 & & \\
\hline Williams & 1 & & \\
\hline Total & 22 & & \\
\hline \multicolumn{2}{|l|}{ Non-syndromic } & & \\
\hline Multisuture & 15 & & \\
\hline \multicolumn{4}{|l|}{ Gender } \\
\hline Male & 24 & \multirow[t]{2}{*}{-} & \multirow[t]{2}{*}{-} \\
\hline Female & 13 & & \\
\hline $\begin{array}{l}\text { Age at first } \\
\text { presentation (months) }\end{array}$ & 37 & $3.8(1.5,21.1)$ & $0.1-59.1$ \\
\hline $\begin{array}{l}\text { Age at first sPVE } \\
\text { surgery (months) }\end{array}$ & 37 & $35.5(9.6,58.6)$ & $2.1-85.2$ \\
\hline $\begin{array}{l}\text { Age at first pre-ICP } \\
\text { clinical exam (months) }\end{array}$ & 37 & $52.8(29.8,71.0)$ & $4.2-152.7$ \\
\hline $\begin{array}{l}\text { Age at first ICP } \\
\text { assessment (months) }\end{array}$ & 37 & $55.7(31.5,73.3)$ & $4.4-154.1$ \\
\hline $\begin{array}{l}\text { Age at final clinical } \\
\text { exam (months) }\end{array}$ & 36 & $88.7(66.6,122.4)$ & $20.3-185.9$ \\
\hline $\begin{array}{l}\text { Total follow-up } \\
\text { (months) }\end{array}$ & 36 & $80.9(53.7,104.7)$ & $18.5-156.2$ \\
\hline
\end{tabular}

$I Q R$ interquartile range, $s P V E$ spring-assisted posterior vault expansion.

defined as $\geq 0.30$ logMAR in the better eye [20]. Two patients (5.6\%) had visual impairment and two patients (5.6\%) had severe visual impairment, defined as 1.0-2.0 $\log M A R$ and $\geq 2.00 \log M A R$ in the better eye, respectively [23]. Non-syndromic patients had better final BCVA (median: 0.20 logMAR; IQR: 0.36 ; range: -0.06 to 1.45 ) as compared to syndromic patients (median: 0.30 logMAR; IQR: 0.43; range: -0.04 to 2.70 ). This difference was statistically significant (Mann-Whitney $U$ test; $z$-score $2.11 ; p=0.03$ ).

\section{Additional outcome measures}

There was a high prevalence of amblyogenic factors in this cohort, including manifest strabismus (52.8\%), astigmatism >1.5 D (34.4\%) and V-pattern (25\%). Supplementary Table 2 (Supplementary Material) provides a full breakdown of amblyogenic factors.

\section{DISCUSSION}

To the best of our knowledge, this is the largest study of patients with craniosynostosis undergoing gold standard invasive ICP monitoring with serial ophthalmological examinations to understand how these measurements change with IH. Fundoscopy demonstrated low sensitivity $(32.1 \%$; 95\% Cl: 15.9-52.4) and high specificity $(100 \%$; $95 \% \mathrm{Cl}: 59.0-100)$ for $\mathrm{IH}$. Isolated VEPs demonstrated $58.3 \%$ sensitivity $(95 \% \mathrm{Cl} 36.6-77.9)$ and $83.3 \%$ specificity (95\% Cl: 35.9-99.6). Where longitudinal deterioration was subtle/suspicious (cumulative score $\geq 1$ ), sensitivity increased to $70.6 \%(95 \% \mathrm{Cl} 44.0-89.7)$, while specificity decreased to $60 \%$ (95\% $\mathrm{Cl}$ : 14.7-94.7\%). Where longitudinal deterioration was clinically 


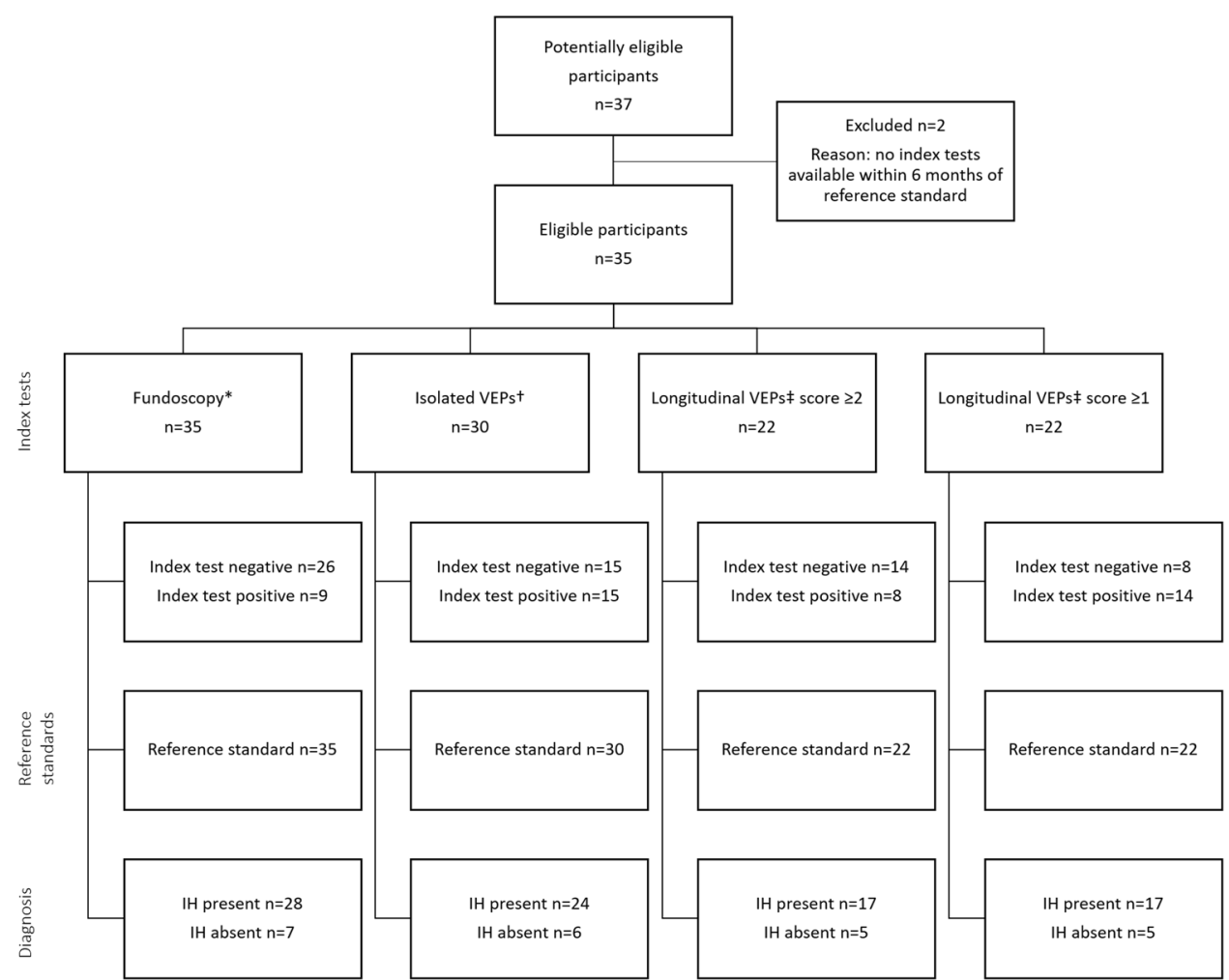

Fig. 1 STARD patient flowchart. * Fundoscopy performed within 6 months of ICP assessments. ${ }^{\dagger}$ Isolated VEPs recorded at visit immediately prior to and within 6 months of ICP assessment. ${ }^{\ddagger}$ Longitudinal VEPs recorded as per isolated, plus two preceding visits; thresholds for deterioration defined as cumulative score of $\geq 2$ and $\geq 1$. IH intracranial hypertension, STARD standards for reporting diagnostic accuracy studies, VEP visual evoked potentials.

Table 3. $2 \times 2$ contingency tables.

\begin{tabular}{|c|c|c|c|c|c|c|c|}
\hline \multirow[t]{2}{*}{$2 \times 2$ tables } & \multicolumn{2}{|l|}{ IH } & \multirow[t]{2}{*}{ Sensitivity $(95 \% \mathrm{Cl})$} & \multirow[t]{2}{*}{ Specificity $(95 \% \mathrm{Cl})$} & \multirow[t]{2}{*}{ PPV (95\% Cl) } & \multirow[t]{2}{*}{ NPV $(95 \% \mathrm{Cl})$} & \multirow{2}{*}{$\begin{array}{l}\text { Diagnostic accuracy } \\
(95 \% \mathrm{Cl})\end{array}$} \\
\hline & Present & Absent & & & & & \\
\hline \multicolumn{3}{|c|}{ Fundoscopy ${ }^{\mathrm{a}}(n=35)$} & $32.1 \%(15.9-52.4)$ & $100 \%(59.0-100)$ & $100 \%(70.1-100)$ & $26.9 \%(22.2-32.2)$ & $45.7 \%(28.8-63.4)$ \\
\hline Non-PE & 19 & 7 & & & & & \\
\hline \multicolumn{3}{|c|}{ Isolated VEPs ${ }^{\mathrm{b}}(n=30)$} & $58.3 \%(36.6-77.9)$ & $83.3 \%(35.9-99.6)$ & $93.3 \%(69.4-98.9)$ & $33.3 \%(21.6-47.5)$ & $63.3 \%(43.9-80.1)$ \\
\hline Abnormal & 14 & 1 & & & & & \\
\hline Abnormal & 8 & 0 & & & & & \\
\hline Normal & 9 & 5 & & & & & \\
\hline \multicolumn{3}{|c|}{ Longitudinal VEPs ${ }^{c}$ score $\geq 1 \quad(n=22)$} & $70.6 \%(44.0-89.7)$ & $60.0 \%(14.7-94.7)$ & $85.7 \%(66.3-94.8)$ & $37.5 \%(17.7-62.6)$ & $68.2 \%(45.1-86.1)$ \\
\hline Abnormal & 12 & 2 & & & & & \\
\hline Normal & 5 & 3 & & & & & \\
\hline
\end{tabular}

$C l$ confidence interval, IH intracranial hypertension, non-PE non-papilloedematous, $P E$ papilloedematous, NPV negative predictive value, $P P V$ positive predictive value, VEP visual evoked potentials.

${ }^{\text {a}}$ Fundoscopy performed within 6 months of ICP assessments.

bIsolated VEPs recorded at visit immediately prior to and within 6 months of ICP assessment.

'Longitudinal VEPs recorded as per isolated, plus two preceding visits; thresholds defined as cumulative score of $\geq 2$ and $\geq 1$.

significant (cumulative score $\geq 2$ ), sensitivity decreased to $47.1 \%$ (95\% Cl 23.0-72.2), while specificity increased to $100 \%$. Papilloedema resolved in all nine affected patients by final examination. Median final BCVA was 0.24 logMAR (IQR: 0.51 ; range: -0.06 to 2.7 ). On final visit, the majority of children (72.2\%) achieved UK driving standard vision, defined as $\geq 0.30 \log M A R$ in the better eye [20].
There was a significant prevalence of amblyogenic risk factors, as expected in this patient population $[14,24]$.

\section{Ophthalmological monitoring}

This study has highlighted the role of ophthalmological monitoring to detect IH and promptly refer for sPVE surgery, albeit no method 
A.

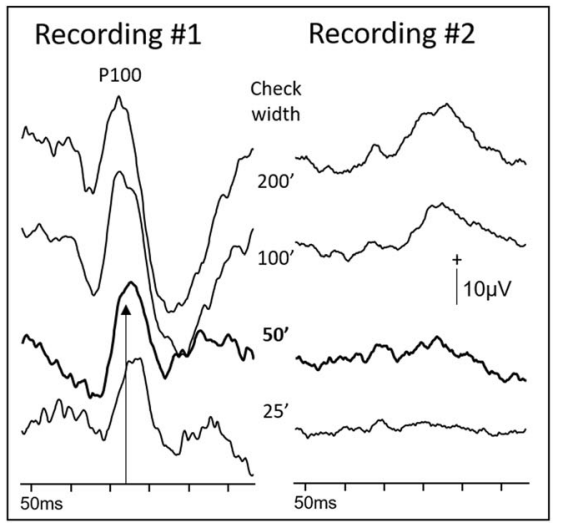

B.

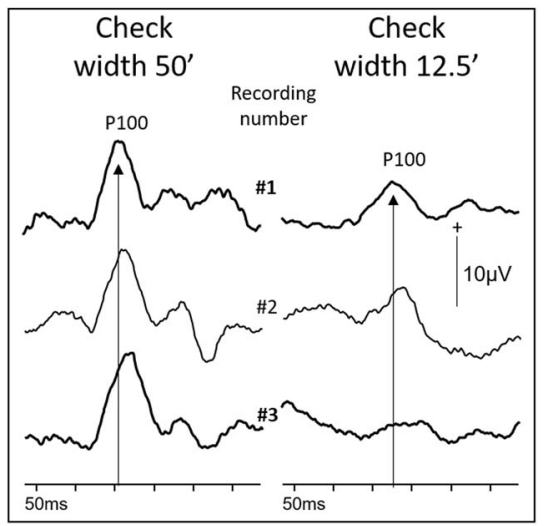

Fig. 2 prVEP waveforms from two patients demonstrating deterioration over time. A Isolated analysis reveals a marked amplitude reduction to prVEPs produced to a range of different check widths, including abnormal prVEPs to 50 ' checks (in bold) between recording \#1 and \#2. At baseline, recording \#1 the 50' check P100 latency is equivocal, borderline for age, but amplitudes are normal. B Longitudinal analysis displays group averaged prVEPs recorded to the ISCEV large $\left(50^{\prime}\right)$ and small $\left(12.5^{\prime}\right)$ checks on three consecutive visits. Deterioration is evident as an increasing delay in P100 latency to small checks between recordings \#1 and \#2: a delay of $+4 \mathrm{~ms}$ to $50^{\prime},\left(+9 \mathrm{~ms}\right.$ for $25^{\prime}$ not shown) and $+12 \mathrm{~ms}$ for $12.5^{\prime}$ check widths. Further deterioration between recordings \#2 and \#3 is noted as reduction in P100 amplitude to the small checks $12.5^{\prime}$ (and 6.25' not shown). prVEP pattern reversal visual evoked potential, ISCEV International Society for Clinical Electrophysiology of Vision.

displayed $100 \%$ sensitivity. When used as part of a multidisciplinary approach, ophthalmological evaluation may prompt neurosurgical evaluation and timely surgical intervention as appropriate.

Our study found that fundoscopic observation of papilloedema reliably indicated $\mathrm{IH}$, but its absence did not exclude $\mathrm{IH}$. PrVEPs were more helpful in detecting IH. PrVEPs had a high sensitivity of $70.6 \%$ for detecting $\mathrm{IH}$ when based on the stability of three serial VEPs. It is an important consideration that isolated VEP abnormalities observed within six months of $\mathrm{IH}$ detection only had a moderate sensitivity (58.3\%), as clinically we may observe a deterioration in VEPs that remain within normal limits; therefore, longitudinal monitoring is essential in patients at risk of $\mathrm{IH}$. The higher sensitivity of longitudinal VEPs most likely reflects the wide range of check widths used for VEP testing, which includes, but extends the ISCEV standard, and use of the individual patient as their own control. Often the earliest change is noted in the prVEP produced by the smallest check widths in the previous recording of the individual's VEPs, suggesting a range of check widths is beneficial in monitoring $\mathrm{IH}$ [18]. The benefits of prVEPs over other methods of ophthalmic monitoring, such as VA or fundoscopy, in young children is that their values typically reach within $10 \%$ of adult values by 6 months of age [25]. Therefore, where fundoscopy is subjective and VA matures over a longer time period requiring different techniques of assessment, the VEP can be used throughout childhood as a measurement of visual pathway stability. Regarding whether VEP reflects IH versus optic atrophy, we feel there may be a complex interplay between both; we can observe improvement of VEPs following surgical intervention, suggesting $\mathrm{IH}$ rather than atrophy; however, some do not improve to the baseline level after deterioration, perhaps reflecting a degree of optic atrophy. Finally, it should be noted that VEP results should be interpreted relative to locally derived VEP reference ranges, as these are dependent on the local stimulus and recording parameters of each laboratory.

\section{Comparison with existing literature}

Visual evoked potentials. The VEP grading criteria by Thompson et al. [13] were modified in this study according to the experience of our laboratory since the initial publication of these criteria. We have observed that 'giant' (i.e., atypically large) VEP amplitudes can be associated with patients with $\mathrm{IH}$. Furthermore, early peak times suggest enhancement of the paramacular pattern VEP contributions, which would suggest macular pathway dysfunction
- something we also note clinically. Our finding of sensitivity $70.6 \%$ is similar to recent studies by Haredy et al. $[26,27]$ who conducted a retrospective study in a smaller sample of 13 children with craniosynostosis, demonstrating a sensitivity of $71.4 \%$ for detection of $\mathrm{IH}$, but with $100 \%$ specificity compared to $60.0 \%$ specificity in our group of 22 patients. Using serial prVEPs in their later publication [27], eight of nine patients with invasively detected IH had abnormal VEPs. Our study used a range of check widths and serial prVEP recordings and, with the findings of Haredy et al. [27], support the improved sensitivity afforded by the longitudinal change to small check widths compared to a prVEP to a single check width alone. PrVEPs produced by small check widths are altered early in optic nerve dysfunction in other diseases [25]. Our findings suggested that a range of check widths for serial prVEP monitoring of patients at risk of $\mathrm{IH}$ is particularly useful. These study findings corroborate the benefits of prVEPs to assess the functional integrity of the macular pathway, which may be affected in early $\mathrm{IH}$, rather than depending upon the subjective observation of frank papilloedema, which is a specific manifestation of IH. The VEP is a signal detected from the cortex after traversing the entirety of the macular pathway. As such, it is susceptible to changes in ICP; moreover, prVEPs to large stimuli occupy a larger field than VA measurements. The VEP can be attenuated with reduced contrast sensitivity and by field changes, whereas VA may remain insensitive as it is a high-contrast test influenced by cognitive association. Further work is needed to explore the role of VEP monitoring in other causes of paediatric $\mathrm{IH}$.

We used both quantitative and qualitative interpretation of VEPs to determine stability in our cohort. In some circumstances quantitative values would indicate a deterioration but when overlaying serial waveforms to compare morphology, it became more evident that measurements could be influenced by background noise or be inconsistent across different check widths. This may explain the lower sensitivity in our series than reported by Haredy et al.

The mechanism behind the earlier change in prVEPs to smaller checks relative to larger checks or VA measurements is likely complex, but probably reflects some multiplicity of reduced contrast sensitivity, modified spatial tuning function due to subtle changes in axonal physiology in the optic nerve that are not reflected in measurements of recognition VA, which is a highcontrast measurement influenced by higher cortical processing. These findings corroborate the benefits of prVEPs in 
multidisciplinary assessments of craniofacial children as they assess the entirety of the macular pathway that may become dysfunctional or modified from changes in early $\mathrm{ICH}$, rather than the observation of papillodema alone that is manifestation of frank $\mathrm{ICH}$ and subjective in nature.

Fundoscopy. Tuite et al. [12] conducted a large study of 122 children with craniosynostosis who underwent fundoscopy and ICP monitoring. They found excellent specificity (98\%) for papilloedema on fundoscopy and raised ICP. Sensitivity of fundoscopy was age dependent: in children over 8 years old, sensitivity was $100 \%$, whereas in children under 8 years old, it was only $22 \%$. These were similar to our findings of $32.1 \%$ sensitivity and $100 \%$ specificity. Another study by Judy et al. [28] found sensitivity $17 \%$ and specificity $100 \%$ for papilloedema on fundoscopy and $\mathrm{IH}$, albeit only 4 of 45 patients (8.9\%) had papilloedema on fundoscopy. Tuite et al. [12] and Judy et al. [28] defined IH as $\geq 15 \mathrm{mmHg}$, whereas our unit now defines this as $\geq 20 \mathrm{mmHg}$. If we had adopted the former definition of $\mathrm{IH}$ in our study, only one child would have been reclassified from normal to raised ICP, but interestingly they had normal fundoscopy in all clinical visits and good final BCVA meeting UK driving standards.

\section{Strengths and limitations}

Whilst this is perhaps the largest report of ophthalmic monitoring findings in SPVE patients with craniosynostosis undergoing invasive ICP monitoring, the sample size relative to the prevalence of these disorders still needs exploring; this may not be achieved without prospective study against patients with ICP monitoring, as our sample was possibly biased to perform ICP monitoring in those under clinical suspicion or high risk. However, a substantial proportion (20\%) of ICP assessments was deemed as normal. More detailed grading of optic disc swelling, such as that offered by the Modified Frisén Scale [29], may have provided opportunity for more detailed description of disc appearance, but this was not possible due to the retrospective nature of this study.

VEP analysis and grading were performed by masked reviewers (ORM and DAT) to avoid experimenter bias. Perhaps one of the main limitations highlighted by this study was that VEPs may be more sensitive where clinicians are suspicious but uncertain of deterioration, for example, due to poor test compliance. VEPs with a cumulative score of 1 had a higher sensitivity than those with definitive deterioration (i.e., score of 2 or above). This is a clinically challenging circumstance, to identify where VEPs have deteriorated, but at the expense of higher sensitivity the specificity reduces also. The complementarity of multidisciplinary assessment has value here. Future work may hope to address this knowledge gap to identify coefficients of variability for paediatric VEP data through maturation to inform future analyses.

Different vision testing methods were used in different children, which may provide diverse estimates of true VA, albeit the majority were able to perform logMAR chart testing at final visit. Refractive data were unavailable for five patients, as these refractions were performed by local optometrists. Optical coherence tomography (OCT) was not possible in young infants in this cohort. However, our unit has recently adopted handheld OCT imaging for young infants with craniosynostosis, using a recently published image acquisition protocol [30], as well as fundus photography and B-scan ultrasound wherever possible.

\section{Conclusion}

This study demonstrated the role of fundoscopy and VEPs in a cohort of children with craniosynostosis undergoing SPVE and ICP monitoring. Fundoscopic observation of papilloedema reliably indicated $\mathrm{IH}$, but its absence did not exclude IH. VEPs demonstrated higher sensitivity in this study, but at the expense of specificity depending on method of analysis. Final visual outcomes were generally good with the majority of children achieving UK driving standard vision, but visual morbidity and amblyogenic factors remain substantial and further work is required to optimise clinical decision making and management of craniosynostosis.

\section{Summary}

What was known before

- Craniosynostosis is associated with intracranial hypertension, which can manifest clinically as papilloedema.

- There is a high prevalence of abnormal pattern reversal visual evoked potentials in children with craniosynostosis.

What this study adds

- Papilloedema present on fundoscopy reliably indicates intracranial hypertension in young children with craniosynostosis, but its absence does not exclude it.

- Monitoring of visual evoked potentials can be valuable in detecting intracranial hypertension in children with craniosynostosis. Longitudinal interpretation can boost sensitivity for detecting intracranial hypertension in craniosynostosis, at the expense of specificity.

\section{REFERENCES}

1. Renier D, Sainte-Rose C, Marchac D, Hirsch JF. Intracranial pressure in craniostenosis. J Neurosurg. 1982;57:370-7.

2. Cornelissen $M$, Ottelander BD, Rizopoulos $D$, et al. Increase of prevalence of craniosynostosis. J Craniomaxillofac Surg. 2016;44:1273-9.

3. Wilkie AOM, Johnson D, Wall SA. Clinical genetics of craniosynostosis. Curr Opin Pediatr. 2017:29:622-8

4. Bannink N, Nout E, Wolvius EB, Hoeve HL, Joosten KF, Mathijssen IM. Obstructive sleep apnea in children with syndromic craniosynostosis: long-term respiratory outcome of midface advancement. Int J Oral Maxillofac Surg. 2010;39:115-21.

5. Tamburrini G, Caldarelli M, Massimi L, Santini P, Di, Rocco C. Intracranial pressure monitoring in children with single suture and complex craniosynostosis: a review. Childs Nerv Syst. 2005;21:913-21.

6. Taylor WJ, Hayward RD, Lasjaunias P, Britto JA, Thompson DN, Jones BM, et al. Enigma of raised intracranial pressure in patients with complex craniosynostosis: the role of abnormal intracranial venous drainage. J Neurosurg. 2001;94:377-85.

7. Abu-Sittah GS, Jeelani O, Dunaway D, Hayward R. Raised intracranial pressure in Crouzon syndrome: incidence, causes, and management. J Neurosurg Pediatr. 2016;17:469-75.

8. Thompson DN, Harkness W, Jones B, Gonsalez S, Andar U, Hayward R. Subdural intracranial pressure monitoring in craniosynostosis: its role in surgical management. Childs Nerv Syst. 1995;11:269-75.

9. Thompson DN, Malcolm GP, Jones BM, Harkness WJ, Hayward RD. Intracranial pressure in single-suture craniosynostosis. Pediatr Neurosurg. 1995;22:235-40.

10. Ramdat Misier KRR, Breakey RWF, Caron CJJM, Schievano S, Dunaway DJ, Koudstaal MJ, et al. Correlation of intracranial volume with head surface volume in patients with multisutural craniosynostosis. J Craniofac Surg. 2020;31:1445-8.

11. Breakey W, Knoops $P$, van de Lande LS, Borghi A, Papaionnou A, O'Hara J, et al. S2-05 Session 2: posterior vault distraction volumetric changes of grey matter, white matter and cerebrospinal fluid following 87 posterior vault expansion cases in patients with craniosynostosis. Plastic and Reconstructive Surgery - Global Open; 2019.7.

12. Tuite GF, Chong WK, Evanson J, Narita A, Taylor D, Harkness WF, et al. The effectiveness of papilledema as an indicator of raised intracranial pressure in children with craniosynostosis. Neurosurgery. 1996;38:272-8.

13. Thompson DA, Liasis A, Hardy S, Hagan R, Hayward RD, Evans RD, et al. Prevalence of abnormal pattern reversal visual evoked potentials in craniosynostosis. Plast Reconstr Surg. 2006;118:184-92.

14. Hinds AM, Thompson DA, Rufai SR, Weston K, Schwiebert K, Panteli V, et al. Visual outcomes in children with syndromic craniosynostosis: a review of 165 cases. Eye (Lond). 2021. https://doi.org/10.1038/s41433-021-01458-5.

15. Tuite GF, Evanson J, Chong WK, Thompson DN, Harkness WF, Jones BM, et al. The beaten copper cranium: a correlation between intracranial pressure, cranial 
radiographs, and computed tomographic scans in children with craniosynostosis. Neurosurgery. 1996;39:691-9.

16. Hayward R, Britto JA, Dunaway D, Evans R, Jeelani Nu, Thompson D. Raised intracranial press ure and nonsyndromic sagittal craniosynostosis. J Neurosurg Pediatr. 2015;16:346-8.

17. Bossuyt PM, Reitsma JB, Bruns DE, Gatsonis CA, Glasziou PP, STARD Group. STARD 2015: an updated list of essential items for reporting diagnostic accuracy studies. BMJ. 2015;351:h5527.

18. Odom JV, Bach M, Brigell M, Holder GE, McCulloch DL, International Society for Clinical Electrophysiology of Vision. ISCEV standard for clinical visual evoked potentials: (2016 update). Doc Ophthalmol. 2016;133:1-9.

19. Hayward R, Britto J, Dunaway D, Jeelani O. Connecting raised intracranial pressure and cognitive delay in craniosynostosis: many assumptions, little evidence. J Neurosurg Pediatr. 2016;18:242-50.

20. Gov.uk. Driving eyesight rules. https://www.gov.uk/driving-eyesight-rules. Accessed 8 Oct 2021

21. Donahue SP, Arnold RW, Ruben JB, AAPOS Vision Screening Committee. Preschool vision screening: what should we be detecting and how should we report it? Uniform guidelines for reporting results of preschool vision screening studies. J AAPOS. 2003;7:314-6.

22. Lange CAK, Feltgen N, Junker B, Schulze-Bonsel K, Bach M. Resolving the clinical acuity categories "hand motion" and "counting fingers" using the Freiburg Visual Acuity Test (FrACT). Graefes Arch Clin Exp Ophthalmol. 2009;247:137-42

23. Royal National Institute of Blind People. The criteria for certification. https://www. rnib.org.uk/eye-health/registering-your-sight-loss/criteria-certification. Accessed 21 Jan 2021.

24. Khan SH, Nischal KK, Dean F, Hayward RD, Walker J. Visual outcomes and amblyogenic risk factors in craniosynostotic syndromes: a review of 141 cases. $\mathrm{Br}$ J Ophthalmol. 2003;87:999-1003.

25. Thompson DA, Kriss A, Taylor D, Russell-Eggitt I, Hodgkins P, Morgan G, et al. Early VEP and ERG evidence of visual dysfunction in autosomal recessive osteopetrosis. Neuropediatrics. 1998;29:137-44.

26. Haredy MM, Liasis A, Davis A, Koesarie K, Fu V, Losee JE, et al. Serial, visually-evoked potentials for the assessment of visual function in patients with craniosynostosis. J Clin Med. 2019;8:1555.

27. Haredy MM, Liasis A, Fu V, Davis A, Pollack IF, Losee JE, et al. Serial visual evoked potentials in patients with craniosynostosis and invasive intracranial pressure monitoring. Plast Reconstr Surg. 2019;144:446e-452e.

28. Judy BF, Swanson JW, Yang W, Storm PB, Bartlett SP, Taylor JA, et al. Intraoperative intracranial pressure monitoring in the pediatric craniosynostosis population. J Neurosurg Pediatr. 2018;22:475-80

29. Scott CJ, Kardon RH, Lee AG, Frisén L, Wall M. Diagnosis and grading of papilledema in patients with raised intracranial pressure using optical coherence tomography vs clinical expert assessment using a clinical staging scale. Arch Ophthalmol. 2010;128:705-11.

30. Rufai SR, Thomas MG, Purohit R, Bunce $C$, Lee $H$, Proudlock FA, et al. Can structural grading of foveal hypoplasia predict future vision in infantile nystagmus?: a longitudinal study. Ophthalmology. 2020;127:492-500.

\section{ACKNOWLEDGEMENTS}

We would like to thank all the patients and families who took part in this study.

\section{AUTHOR CONTRIBUTIONS}

SRR: conceptualisation, methodology, formal analysis, investigation, writing-original draft, data curation, visualisation, project administration, funding acquisition. ORM: conceptualisation, methodology, validation, formal analysis, investigation, writingoriginal draft, visualisation. DAT: methodology, formal analysis, investigation, writing -review and editing, supervision. LSvdL: investigation, data curation, writingreview and editing. RWB: investigation, data curation, writing-review and editing. $\mathrm{CB}$ : methodology, validation, formal analysis, writing-review and editing, supervision, funding acquisition. FAP: methodology, validation, formal analysis, writingreview and editing, visualisation, supervision, funding acquisition. VP: investigation, writing-review and editing. KS: investigation, writing-review and editing. SM: investigation, writing - review and editing. IG: methodology, writing-review and editing, supervision, funding acquisition. DJD: methodology, investigation, writingreview and editing. $\mathrm{RH}$ : methodology, writing-review and editing, supervision. RB: conceptualisation, methodology, investigation, writing-review and editing, project administration, supervision, funding acquisition. NuOJ: conceptualisation, methodology, investigation, writing-review and editing, data curation, supervision, project administration, funding acquisition.

\section{FUNDING}

SRR is funded by a National Institute for Health Research (NIHR) Doctoral Fellowship Award. This work is supported by the NIHR Great Ormond Street Hospital Biomedical Research Centre. This paper presents independent research funded by the National Institute for Health Research (NIHR). The views expressed are those of the author(s) and not necessarily those of the NHS, the NIHR or the Department of Health and Social Care.

\section{COMPETING INTERESTS}

The authors declare no competing interests.

\section{ADDITIONAL INFORMATION}

Supplementary information The online version contains supplementary material available at https://doi.org/10.1038/s41433-021-01839-w.

Correspondence and requests for materials should be addressed to Noor ul Owase Jeelani.

Reprints and permission information is available at http://www.nature.com/ reprints

Publisher's note Springer Nature remains neutral with regard to jurisdictional claims in published maps and institutional affiliations.

(i) Open Access This article is licensed under a Creative Commons Attribution 4.0 International License, which permits use, sharing, adaptation, distribution and reproduction in any medium or format, as long as you give appropriate credit to the original author(s) and the source, provide a link to the Creative Commons license, and indicate if changes were made. The images or other third party material in this article are included in the article's Creative Commons license, unless indicated otherwise in a credit line to the material. If material is not included in the article's Creative Commons license and your intended use is not permitted by statutory regulation or exceeds the permitted use, you will need to obtain permission directly from the copyright holder. To view a copy of this license, visit http://creativecommons. org/licenses/by/4.0/.

(c) The Author(s) 2021 